\title{
The Spatio-temporal Dynamics of Systemic Thinking
}

\author{
Gaëlle Vallée-Tourangeau ${ }^{1} \&$ Frédéric Vallée-Tourangeau ${ }^{1}$
}

\begin{abstract}
${ }^{1}$ Kingston University London
Recent developments in cognitive science call for a reconceptualization of cognition as emerging in a system that encompasses the brain and the body in situ, and rejects the classical view that cognition is a fundamentally cerebral activity based on the rule-based processing of symbols represented in the mind. A radical anti-thesis to the classical information-processing approach goes as far as positing that cognition emerges directly from people's actions in the world. This view comes with two corollaries. The first corollary is that representations are unnecessary to explain complex behaviours, which can be understood from the study of the dynamic relation between an agent and her environment. The second corollary is that there is a spatio-temporal dimension to cognition as it emerges from a continuous and fluid coupling of neural and physical activity. In this paper, we discuss the implications of the ecological view of cognition for higher cognitive functions such as problem-solving, judgement, and decision-making. We argue that a radical departure from the classical information-processing model is untenable, notably because higher-level cognition is fundamentally representation-based, thus rejecting the first corollary of the radical embodiment approach. However, we also argue that classical accounts of thinking put too great an emphasis on the role of internal representations and mental processing. This obscures the symbiotic relationship between thinking and acting and the role of spatio-temporal dynamics and ecological affordances on thinking, thus embracing the second corollary of a systemic view of cognition. Finally, we discuss current ecological accounts of higher cognition and show that they are, at best, interactional. To fully understand how people think, solve problems, and make decisions, we need to break from the traditional conception of thinking activities sequestered in a static mind, and instead study how thinking emerges in ecological space and ecological time from the transactional flow of action and representational opportunities outcropping from a dynamic agent-environment interface.
\end{abstract}

Keywords: decision-making; distributed cognition; dynamics system; embodied cognition; grounded cognition; problem-solving; radical embodiment; representations; systemic cognition; thinking

Recent developments in cognitive science call for a reconceptualization of cognition as emerging in a system that encompasses the brain and the body in situ, and rejects the classical view that cognition is a fundamentally cerebral activity based on the rule-based processing of symbols represented in

Gaëlle Vallée-Tourangeau, Department of Management, Kingston University, Kingston-upon-Thames. UNITED KINGDOM, KT2 7LB. Frédéric Vallée-Tourangeau, Department of Psychology, Kingston University, Kingston-upon-Thames. UNITED KINGDOM, KT1 2EE. This is an Accepted Manuscript of an article published by Imprint Academic in Cybernetics $\mathcal{E}$ Human Knowing on 1 January 2014, available online: https://www.ingentaconnect. com/content/imp/chk/2014/00000021/F0020001/art00009. Correspondence concerning this article should be addressed to either Gaëlle Vallée-Tourangeau, g.vallee-tourangeau@kingston.ac.uk, or Frédéric Vallée-Tourangeau, f.vallee-tourangeau@kingston.ac.uk.

Correspondence concerning this article should be addressed to Gaëlle Vallée-Tourangeau, Department of Management, Kingston University, Kingston-upon-Thames. UNITED KINGDOM, KT2 7LB. E-mail: g.vallee-tourangeau@kingston.ac.uk the mind. A radical anti-thesis to the classical informationprocessing approach goes as far as positing that cognition emerges directly from people's actions in the world. This view comes with two corollaries. The first corollary is that representations are unnecessary to explain complex cognition, which can be understood from the study of the dynamic relation between an agent and her environment. The second corollary is that there is a spatio-temporal dimension to cognition as it emerges from a continuous and fluid coupling of neural and physical activity. In this paper, we discuss the implications of the ecological view of cognition for higher cognitive functions such as problem-solving, judgement, and decisionmaking. We argue that a radical departure from the classical information-processing model is untenable to understand how individuals think, notably because thinking is fundamentally representation-based, thus rejecting the first corollary of the radical embodiment approach. However, we also argue that classical accounts of thinking put too great an emphasis on the role of internal representations and mental processing. This obscures the symbiotic relationship between thinking and acting. By contrast, we argue that spatio-temporal dynamics and ecological affordances play a fundamental role in 
thinking, thus embracing the second corollary of a systemic view of cognition. Finally, we discuss current ecological accounts of higher cognition and show that they are, at best, interactional. To fully understand how people think, solve problems, and make decisions, we need to break from the traditional conception of thinking activities sequestered in a static mind, and instead study how cognition emerges in ecological space and ecological time.

\section{Cognition Is an Emergent Property of Dynamical Systems}

\section{The missing part of the Turing machine}

Thinking is an elusive concept. Classically, human thinking has been conceived as the mental process of pondering on, or reasoning about, ideas and opinions produced by the mind. Thinking is therefore a cognitive activity, where cognition is the process of acquiring knowledge and understanding. Most psychologists view cognition as the product of a "Turing Machine", named after the British mathematician who first imagined it. Turing (1936) compared a man to a computing machine, endowed with an initial configuration or "state of mind", a limited set of operations that he can apply to information brought to its conscious awareness, and a limited set of procedures stored in memory dictating the order and numbers of operations to be applied. In this model, cognition results from the desultory, stepwise, processing of information inside one's head. The coupling of an individual's state of mind with the value of the information in his conscious awareness at any moment in time determines which procedure should be applied to transform that information. This results in a new state of mind, which can then be coupled with the next piece of information to enter conscious awareness and identify the next procedure to be applied, and so on. The Turing machine metaphor has had a long-lasting impact on cognitive scientists' conception of cognition; they have since sought to discover and model the mental procedures linking informational inputs and outputs. Outside the realms of cognitive psychology, however, philosophers (Clark, 2008), anthropologists (Hutchins, 1995), and cognitive scientists (Kirsh, 2013) have challenged this conception of cognition, arguing instead that cognition emerges in complex systems that include, but cannot be fully specified by, neural computations. Turing's machine was intended to model a mathematician who proceeds towards a computational goal. Yet, it leaves out the person, with her eyes, ears and hands, and the physical world in which she implements those computations, including, for example, her notebook and fountain pen. It leaves out the notebook's annotations, strikeouts, mistakes, drawings, quotes, and so on. It misses out the dead-ends and insights occurring over time. The Turing machine reduces this rich process to the application of pre-determined rules to strings of symbols and dismisses the contribution of the mathematician's interactions in space and time with the material world as mere implementational details. We are left with a sterilised model of human cognition without a human being in it. As such, the Turing machine epitomises models of the abstract computations that result from the processing of symbols, but it is not an adequate model of human cognition because it fails to explain how people's interactions with the material world through their eyes, ears, and hands support the instantiation of these computations (Hutchins, 1995). Not all dissenters agree on what alternative model we should adopt to account for human cognition. Proponents of the distributed cognition approach continue to characterise cognitive processes as involving mechanisms operating upon representations but they reject the assumption that those mechanisms and those representations are necessarily uniquely mental: "Minds are not passive representational engines, whose primary function is to create internal models of the external world." (Hollan, Hutchins, \& Kirsh, 2000, p. 177). So, rather than conceive cognition as emerging from the activity of the brain alone, the distributed cognition approach conceives cognition as emerging from the coordination of people's inner resources and mental processes with the resources present in their immediate material and social environments, and the processes taking place in these environmental spheres. As such, cognition is taken to arise from the transmission, transformation, and coordination of both mental representations (e.g., as people mentally rotate a geometric form) as well as physical ones (e.g., as people physically manipulate geometric forms in a game of Tetris to speed up identification, Kirsh \& Maglio, 1994). Other dissenters, however, are calling for a more radical reconceptualisation of cognition. For proponents of the radical embodiment approach (e.g., Chemero, 2009; Shapiro, 2011; Van Gelder, 1995; Wilson \& Golonka, 2013), the Turing machine is an inadequate model of human cognition, not only because it confines cognitive processes to those located inside the brain, but also because it assumes that cognition emerges from the processing of complex representations. These views come with two corollaries. The first corollary is that representations and complex computations are unnecessary to explain complex behaviours. The second corollary is that there is a spatio-temporal dimension to cognition. We review both corollaries in the next two sections.

\section{Neither representations nor complex computations are necessary to account for cognition}

Classical constructivist theories of perception cast it as a product of mental processes that disambiguate impoverished environmental input (e.g., Gregory, 1980). Gibson (1986) criticised this view for it implicitly models the perceiver as an immobile, passive observer. He argued that perception was not an approximate, mental process; rather, it was a physical 
activity. From this perspective, perceiving organisms have access to a clear, definitive, and direct identification - that is, an identification that is not mediated by a mental representationof environmental stimuli, through the movements of their head and body as well as their actions. Moreover, the purpose of such a direct perception is not to build a passive catalog of stimulus categories. Its purpose is to identify what the environment may "afford"; that is, what opportunities or possibilities for action it offers (Withagen \& Chemero, 2012). Proponents of the radical embodiment approach propose that Gibson's account of perception is applicable to cognition in general, thus recasting cognition as emerging online, in real time, in situ, and from the dynamical and continuous interactions of coupled systems made of brain, body, and world. From this perspective, meaning and knowledge are not invented through mental processes, nor are they mediated by representational states. Instead, they are discovered through the coupling of the cognising agents' activities and the affordances available in their environment (Turvey \& Shaw, 1999; Wilson \& Golonka, 2013). The assumption that knowledge and understanding arise from the coupling of a knowing agent and the environment within which this agent functions calls for a shift in focus from the study of brain activity as a causal determinant of cognition towards the study of the causal interconnections between the agent and its environment. Cognition is assumed to self-organise over time and emerge "live" from perceptionaction couplings, in a nonlinear, thoughtless, but deterministic way. Constrained by their own biological attributes, the characteristics of the tasks they face, and the features of the immediate environment in which they operate, cognising organisms are ultimately channeled into more or less stable attractor states of mind (Thelen, 1992). To understand cognition is, therefore, first to understand what resources are at the disposal of the knowing agent to solve a specific task. These resources may be located in the body, in the environment, and, eventually, in the brain. Second, to understand cognition is to understand how such resources can be assembled to create a dynamical system that evolves over time towards a solution (Wilson \& Golonka, 2013). Numerous examples from robotics and animal cognition show that complex behaviours can arise from the coupling between agents and their environment without the need to use representations or computations as mediators (e.g., see Barrett, 2011). Rat pups, for example, are born blind, deaf and without fur. To survive, they need to stay close to each other to keep their body temperature at a suitable level. Despite their "disabilities," they exhibit a complex and characteristic pattern of behaviours known as "thigmotaxic behaviours" and which include wall following, corner burrowing and huddling. May et al. (2006) were able to reproduce these behaviours in robot rats without sensors but simply programmed to select randomly from a limited number of movements (stop, move forward in a number of direction or move backward in a couple of directions) every two seconds. The key feature of these roborats was that their body was morphologically similar to that of real rats, and importantly, featured a pointy nose. Once they encountered a wall, the moving robot-rats' pointy nose caused them to slide alongside it until they reached a corner, at which point they became stuck. As more robot-rats joined in, they all ended up huddling in a corner. This example illustrates how complex pattern of behaviours may emerge without the need for mental "action plans". None of the robot-rats intended to huddle. Instead, their behaviour emerged from the interaction between their inner mechanisms (i.e., the different built-in types of motions available to them), their body (i.e., the shape of their nose), and the characteristics of their immediate environment (i.e., featuring walls and corners).

\section{Cognition results from a temporally continuous flow of activity}

A second corollary of the conception of cognition as emerging in real time from the continuous interactions of coupled systems made of brain, body, and world concerns the manner in which cognition evolves in time. The Turing machine metaphor for cognition suggests that cognitive processes unfold in a formally-specifiable sequence of discrete events. The order of these events is assumed to be dictated by a stored algorithm controlling the sequence of steps required to transform an informational input into an output. From this perspective, change is predictable and timing is irrelevant. In contrast, timing becomes an essential feature of cognitive systems if one views cognition as emerging from the realtime coupling of brain and body activities in the environment. The coupling of brain, body and environment in a cognitive system results in simultaneous unfoldings. Brain activity is changing while the knowing agent acts in the world while the world is changing. Brain activity, the agent's actions in the environment, and the resulting changes in the landscape are continuously dependent on each other; they co-evolve in a cyclical pattern of causation and coupling. The temporal dimension to the emergence of cognition spans from milliseconds to weeks, months up to millennia. At the millisecond scale, real-time cognition can be shown to evolve through continuous and dynamic changes rather than "as a staccato series of abstract computer-like symbols" (Spivey \& Dale, 2006, p. 207). EEG studies of insight in remote-associate tasks do not reveal a discrete, sudden, "epiphanic" Aha! moment, but rather a continuous pattern of activation including prior preparatory brain states (Kounios \& Beeman, 2009). Speech perception (e.g., distinguishing between competing phonemes such as "ba" and "pa"), spoken word recognition (e.g., recognising "candle" instead of "candy") and semantic categorisation (e.g., categorising "whale" as a mammal rather than a fish) are all examples of cognitive processes that do not involve a discrete, stepwise, resolution; rather they involve 
a temporally continuous flow of neural activity that is first attracted to competing "gravitational basins" until some "resolution" occurs, leading to a stable mental state (Spivey \& Dale, 2006). Beyond the millisecond span, similar patterns of temporally continuous behaviours can be observed in infants who learn to reach for hidden objects over time. The classic A-not-B error occurs when 10-months old infants persist in reaching for an old hiding place to retrieve an attractive object after having seen the object being hidden in a new location. Traditional computational accounts for this error propose that it arises as motor experience takes precedence over a conscious representational system where behaviour is controlled by rule-based algorithms (Marcovitch \& Zelazo, 1999). This "error" can be explained without resorting to a representational system. Thelen, Schöner, Scheier, and Smith (2001) showed that the perseverance error was determined by multiple causes - such as the history of reaching for the original location, the attention-grabbing of the hiding event or of the cover, the delay between the hiding event and the reaching behaviour, or the approach to reach-all potentially interacting over nested timescales. The error is not a failure of motor control planning at the neuronal level; rather it is the product of the interaction, in real-time, between the infant body, its mind, and the activities afforded by its immediate environment. By the age of 12-months, most infants stop persevering in the classical task; however, changing the environment can make the error re-appear in two-year-olds (Butler, Berthier, \& Clifton, 2002). This suggests that cognitive development is not simply the product of brain maturation; it is the emergent product of the continuous unfolding of infants' activities in their environment, where each action sets the stage for the next (Smith \& Thelen, 2003). So, here again, behaviour cannot be accounted for by a timeless, orderly, plan of actions. Instead, time is of the essence: to predict what will happen at time 1 in the brain-body-world system, you need to know where everyone and everything were at, at time 0 . This is true for the trajectory of cognition at the millisecond timescale, at the lifespan timescale, and the argument can be made that this also account for the development of homo sapiens' cognitive capacities over millennia (Malafouris, 2013). To summarise, recent views, ranging from the distributed cognition approach to the radical embodiment approach recast cognition as emerging, in real-time, from the interactions of a knowing organism, acting in, and reacting to its immediate environment. While the radical embodiment approach is arguing that cognition can do without representations, the type of behaviours this approach aims to explain remain basic (usually implicating action-perception loops to guide adaptive movement in simple environments). Whereas one may ponder at the remarkable (in)ability of super-computers to model simple behaviours, it remains that humans are capable of achieving more than those simple behaviours. Humans can play chess, draw inferences, solve problems and make decisions. In the next section, we discuss the challenges these types of activities raise for both the traditional computational approaches as well as the radical embodiment approach.

\section{The Human Brain is not (Only) an Executive Controller of Actions}

\section{Representations are the undeniable substrate of some hu- man cognitive activities}

The assumption that cognition emerges from a system that never represents the world may be tenable from the perspective of robotics or animal psychology; but it is largely untenable from the perspective of cognitive psychology- the branch of psychology that is concerned with how humans acquire, transform, and produce knowledge. Notwithstanding David Hume's (1740/1983, as cited in Turvey \& Shaw, (1999)) touchstone, some cognitive activities are unique to humans and may require an explanatory framework that is inapplicable to other organisms. For a start, there is ample empirical evidence that people represent the meaning of what they have read or heard. For example, Bransford and Franks (1971) showed that, after having studied a set of sentences such as "the ants in the kitchen ate the jelly" (a) and "the ants ate the sweet jelly, which was on the table" (b), people would later be equally likely to recognise an old sentence such as (a) or a new sentence combining propositions from studied sentences, such as (c): "the ants ate the sweet jelly." However, they would not recognise a sentence that contained new units of abstract meaning such as "the ants ate the jelly beside the woods." Such findings strongly suggest people construct a mental representation of the gist meaning of the sentences they read and later rely on those representations to support their recall or recognition. Besides meaning, people also represent and solve problems. Two broad types of problems have been subjected to laboratory scrutiny by psychologists: (i) analytic or transformation problems and (ii) insight problems. Transformation problems are knowledge lean and welldefined problems, presenting an initial situation or start state, and a final situation or goal state. To solve transformation problems, people are assumed to represent the problem's start state and engage in a step-by-step mental transformation of this representation until they reach the problem's goal state. Such transformations occur by applying operators, defined as more or less constrained means of changing one state into another. Simple transformation problems such as the Tower of London have been studied in the lab, primarily to observe, or in neuropsychological cases to diagnose, participants' planning skills and move selection decisions. Insight problems, although they too tend to be knowledge lean, are less easily characterized although all involve an initial inability to envisage the operators one could apply to achieve the goal state. It is this initial impasse that must be overcome for a solution to 
be conceived. Insight problems interest psychologists largely because of the "Aha" phenomenology associated with the sudden eureka clarity with which reasoners envisage the solution. Take the following problem: How do you throw a ping pong ball in such a manner that it comes to a complete stop and reverses direction without coming into contact with anything? An initial representation of the problem involving a horizontal throwing motion will encourage possibly creative but futile solutions. However, representing a vertical throwing motion quickly ushers in a satisfying resolution to this "problem". What interest psychologists are the processes by which the initial representation of the problem is restructured to yield a more productive perspective from which the solution can be conceived easily. Once again, the fact that people can solve both classes of problems, simply from reading a problem statement written on a piece of paper (e.g., Metcalfe \& Wiebe, 1987) suggests that people construct mental representations of problem states and rely on those representations to support their problem solving activities. Representations of meaning and problem states are two among many more examples of higher cognitive activities. Similar arguments can be made with regards to other higher cognitive activities such as mathematical reasoning (e.g., Ashcraft \& Battaglia, 1978), analogical reasoning (e.g., Gick \& Holyoak, 1983), deductive and inductive reasoning (Johnson-Laird, 1983), or judgement and decision-making (Villejoubert \& Mandel, 2002). The fact is that humans are able to think off-line. Put a hungry human in a new maze (e.g., a new city). Typically, he may hunt for a grocery store or restaurant randomly walking along city blocks. He might remember his way around initially through trials and errors but will soon develop a cognitive map of his surroundings. Early orientation behaviours may be closely coupled to perceptual inputs, but soon he will be able to plan his whereabouts or figure out ways to overcome changes in his environment off-line; that is, in the absence of concurrent dynamic interaction. This, as Clark (1997) aptly put it, points to "the difference between inner systems that operate only so as to control immediate environmental interactions and ones that use inner resources to model the world so as to obviate the need for such continual environmental interaction." (p. 464). In other words, cognitive psychology, and more particularly higher cognitive functions, can hardly be conceived as unfolding solely from on-line perception-action couplings, as proponents of the radical embodiment approach sometimes argue. This raise the question of the part, if any, played by on-line perception-action couplings in higher cognition. We address this issue next.

\section{Is there a case for an objectivist account of cognition?}

Cognitive psychologists have traditionally embraced Fodor's (1980) "methodological solipsism", assuming higher cognitive functions take place inside the head, squeezed between perceptual inputs and behavioural outputs. Those who adopt this conception of cognition effectively ostracise the world outside the thinking head, and aim to characterise higher thinking through relatively static mental states and structures. From this perspective, perception-action couplings are not constitutive part of cognition, they are incidental by-products, implementation spandrels, of internal computation. The fact that something that is happening in the environment has some causal influence on cognitive activity does not warrant the conclusion, intracranialists argue, that such an external event is constitutive of cognition. From this perspective, tool use, for instance, is "typically a matter of cognitive processes interacting with portion of the non-cognitive environment" (Adams \& Aizawa, 2009, p. 80). Moreover, the brain constitutes a natural kind and, consequently, its workings can be assumed to be bound upon by natural laws and its functions and processes are multiple but they are homogeneous because they all emerge from the same (neurological) substrate. These facts make a scientific account of brain cognition possible. By contrast, goes the argument, tools are not natural; they are artefactual and disparate. The processes involved in thinking with tools are not homogeneous, they are varied and indefinable; therefore, there cannot be a scientific account of brain-tool cognition that would lead to the discovery of general laws of behaviour. This position, it seems to us, underscores the deficiency of the current research methodology to capture how cognition may emerge from brain, body, and environment interactions rather than a genuine theoretical and ontological impasse. There are many examples throughout history where human understanding of natural phenomena shifted radically through methodological breakthroughs and the accumulation of empirical evidence. The brain itself was once considered such a trivial organ that it was simply removed in the preparation of bodies for mummification in ancient Egypt or conceived as a mere cooler for the heart's passions in Aristotle writings (Finger, 1994). Admittedly facile, this diachronic contrast nevertheless illustrates how our current understanding of the workings of the brain was made possible once scholars believed in the importance of its study, developed and honed methods to study it, and started to accumulate empirical findings. To conclude a priori that the external props and aids are motley and irrelevant to cognition is unwarranted and risks missing a proverbial forest. Until an appropriate methodology is developed for studying how cognition may emerge from the dynamic configuration of extended cognitive systems, and until enough empirical evidence is accumulated, we are bound to see motley. Motley may seemingly preclude a scientific account of how transactions between brains, bodies and environments contribute to the emergence of higher cognition, but it does not necessarily entail that interactivity is devoid of meaningful structures, organizing patterns, and regularity. Only the empirical and scientific study of those systems can provide a meaningful 
answer to this question. This new programme of research may begin with a more qualitative and ethnographic research methodology (see Steffensen, 2013, for an excellent example) but could also borrow from established methods of applied behavior analysis in germane fields of enquiries such as developmental psychology, educational psychology and ethology (Bakeman \& Quera, 2011; Sharpe \& Koperwas, 2003). Such approaches are well poised to offer generalizable conjectures about how new ideas, understanding, and solutions emerge from an ontological substrate woven by interactivity. Note that this is not to say that we should treat artefact materiality as inconsequential: the evolution and transformation of an approximate number sense to exact counting and arithmetic in humans over millennia of cultural evolution is inextricably linked to the development and manufacturing of a wide range of artefacts-clay tokens, so-called "envelopes", impressed tablets, and pictographic tablets (Malafouris, 2013). On the contrary, this is a call to embrace an epistemological approach and an empirical commitment to document how cognitive systems work. A related argument put forward against the proposition that external props and aids are constitutive of human cognitive processing is the fleeting nature of systems where brain and body interact with their immediate environment (Rupert, 2009). Cognitive psychology's aim, admittedly, is to provide a nomothetic account of human cognitive processes through the discovery of general scientific laws that underpin human cognitive activities such as categorisation, memory, judgement and problem-solving, across contexts and situations. By contrast, situated systems where brain, body and environment interact seemingly epitomise the idiographic particular, with its essentially transient and concrete interactions bounded to a specific space and time. So in the face of the motley of environments, artefacts, and localised interaction, the brain appears as the only constant and thus the only suitable unit for studying human cognition. This argument, however, is fallacious. The complex patterns of neural cognitive activity unfolding inside one individual brain, at any point in space and time, are as particular, idiosyncratic and seemingly unruly as the motor activities unfolding outside the brain, at this very moment, in this very situation. Still, neuroscientists can formulate function-to-structure deductive and structure-to-function inductive inferences (Henson, 2005) despite heterogeneous patterns of brain activity. So, once again, the fleetness argument does not hold up as an argument against the possibility of formulating generalizable inductive inferences about the spatio-temporal dynamic regularities of systemic cognition.

To summarise, the fact that individuals can think off-line does not take away the fact that they do not typically do so (Clark, 2010). People think everyday and yet, undeniably rarely come up with solutions to concrete or abstract problems through long streaks of off-line thinking. Instead, most of human thinking emerges online, through physical and verbal interactions with the immediate environment. For example, real-world problem solving is enacted through interactivity with people and things in a physical environment (Cowley \& Nash, 2013; Steffensen, 2013). Scientists use artefacts and models to help them formulate and test hypotheses (Giere \& Moffatt, 2003; Watson, 1968), and people naturally recruit artefacts and tailor their immediate physical environments to augment and transform their problem solving activities. Once one recognises that cognition typically unfolds in a brain-body-environment system, the question of whether artefacts form a constitutive part of human cognition-e.g., to paraphrase Clark and Chalmers (1998), whether Otto's notebook is included in his mind—or the question of whether or not cognitive processing only takes place inside brains both become peripheral to the concerns of the systemic cognitivist. The important issue is no longer where cognitive processing begins and where it ends. The important issue becomes how cognition emerges from the interactions of brain activity, motor actions, and artefacts. Systemic cognitivists do not question whether there can be a scientific study of real-time cognition. Actions are not unbounded: body and environment constraint the interactive trajectory. To adopt a systemic approach to cognition is to reject the a priori assumption that there are no general laws that can account for how cognition emerges in situ, and embark on a scientific journey of discovery, documentation and ultimately theoretical understanding of untapped psychological phenomena.

\section{The Spatio-temporal Dynamics of Systemic Thinking}

\section{Interactivity plays a constitutive role in thinking (and in shaping the world that enacts it)}

As it disregard actions in the world as merely peripheral, traditional cognitive psychology has generally been reluctant to study thinking in problem-solving environments that permit and encourage interactivity (Vallée-Tourangeau \& Villejoubert, 2013). Once that reluctance is shed, and modest efforts go toward implementing interactive thinking environments in the lab, there is much evidence that interactivity substantially transforms problem solving (Vallée-Tourangeau, 2013; ValléeTourangeau, Euden, \& Hearn, 2011). Thus, for example, we observed that interactivity promoted a significantly higher rate of insight solutions in problems for which a false algebraic expression with Roman numerals can be transformed into a true one by moving a single feature of either a numeral or an operator (e.g., how to transform I = II + II into a true expression). Weller, Villejoubert, and Vallée-Tourangeau (2011) demonstrated that participants were more likely to discover the solution (e.g., I = III - II) if the expressions themselves were presented as manipulable three dimensional objectsand participants were invited to manipulate them- than if they were presented on a piece of paper and participants 
announced their solution to the experimenter. Interactivity matters because action-perception cycles quickly unveil a range of potential solutions that help participants home in on the right one. Participants may experience a form of impasse, but the opportunity to manipulate the physical elements of the problem evinces a fluid set of action affordances, which encourage participants to tinker and continue their exploration. In turn, when participants are confronted with an unchanging algebraic expression, the perceptual feedback exerts a strong conceptual pull back to the false formulation: The participants must overcome this perceptual information by simulating the transformations mentally. To be sure, the problem solving exercise exacts a greater toll on working memory in a static than in an interactive condition. But interactivity does not simply augment working memory resources by letting the environment shoulder some of the computational burden; this indeed "hugely downplays what is going on" (Kirsh, 2013, p. 178). Interactivity transforms the terrain of cognition: the thinking that emerges from the intertwining of a reasoner's internal resources and the external artefacts is qualitatively different. Performance on mental arithmetic problems provides another example of how interactivity transforms thinking. Asking college-age students to add a long series of singledigit numbers, without using pen and paper, does not exceed their acquired arithmetic skills. However, a long series of numbers arrayed in a random configuration puts a significant burden on working memory-participants must remember the interim total, what numbers were added, which one should be added next - and counting inaccuracies are likely to arise. Of greater interest, is the strategy employed in this situation: Participants are likely to adopt a simple strategy that conserves working memory resources, scanning single numbers in turn, keeping track of the running total. This slow and methodical strategy underexploits stored knowledge of simple sums which further handicaps the ability to identify congenial groupings and subtotal to enhance the speed and accuracy of the process. The same sum presented in terms of movable number tokens that participants are encouraged to move about will give rise to substantially different counting behavior. As Vallée-Tourangeau and Villejoubert (2013) demonstrated, interactivity fosters more creative and efficient paths to solution in mental arithmetic: participants are less likely to adopt a conservative counting strategy, and better able to identify groupings that facilitate the creation of congenial interim totals (e.g., such as those that divide by 5 without a reminder). Interactivity thus enhances the robustness and efficiency of the "mental" calculation process. Interactivity provides additional working memory resources, but the transformative impact on thinking cannot be predicted by the simple addition of external storage.

\section{Thinking emerges from transactions between online and offline cognition}

Classical accounts of thinking put too great an emphasis on the role of internal representations and mental processing. This is not to say that the impact of the environment has not been featured in such accounts, however. Core cognitive processes have often assumed to result from both inner processes and the situation within which people are embedded while they think (e.g., Neisser, 1967). The world, however, is assumed to be mirrored (more or less accurately) in people's heads and the important question becomes "how an exploitative relationship between mind and environment has implication for the kind of cognitive machinery used by the mind" (Brighton \& Todd, 2009, p. 324). The answer, according to proponents of the ecological rationality approach is that adaptive behaviour emerges when there is a match between information structure in the mind and in the world. Thus, this conception of cognition remain subordinate to the doctrine of animal-environment dualism (Turvey \& Shaw, 1999). It conceives the mind and the environment as disjointed entities and cognition as emerging from a "self-actional" brain, which has evolved its computational mechanisms in symbiosis with nature, and as a result may be more efficient in computing "natural" information (e.g., frequency information rather than probabilistic information; Gigerenzer \& Hoffrage, 1995) but nevertheless computes information in a timeless manner, and independently of its immediate environment. This can be contrasted with a situated and interactional account of cognition, where the immediate environment has a direct impact on what is processed by the brain and how it is processed as well as where, conversely, what is processed by the brain can have a direct impact on the immediate environment (e.g., where the immediate environment is transformed the instantiation of a plan of action initially elaborated in the brain). Such an interactional account of cognition, however, is still limited by a mechanical conception of brain and environment as having independent influences on cognition. Intracranialists will readily grant the importance of the environment in shaping cognition and behavior: "the apparent complexity of our behavior $(\ldots)$ is largely a reflection of the complexity of the environment in which we find ourselves (Simon, 1969, p. 53). But the interaction between two independent entities can be parsed in terms of input-output segments, betraying an insidious dualism. This research programme misses the mark (see also Villejoubert \& Vallée-Tourangeau, Frederic, 2011). The dynamic context in which cognition and behavior emerge reflects transactional forces that coincidentally and reciprocally shape cognition and the world that enacts it. In our laboratory work on problem solving, the proto representations of a solution and the world that in turn reflects it, as well as offering the means to project its potential development, both co-evolve in space and time to a more complex representation 
of the solution. The only research methodology that can capture the genesis of insight in problem solving research is one that examines the thoughts and behaviours of an agent in real time in a dynamic and malleable physical environment. As such, Steffensen's (2013) cognitive event analysis alongside other methods of applied behavior analysis offers a promising compass to guide these future efforts.

\section{Concluding Remarks}

We draw considerable inspiration and guidance from a more radical form of embodied cognition. However, the associated philosophical commitments that jettison mental representations in thinking require a herculean dismissal of voluminous evidence to the contrary. We also argue that traditional research methods in cognitive psychology reflect a dualism that keeps mind and environment in distinct ontological categories. Classical accounts of thinking put to great an emphasis on internal representation and mental processes and ignore the symbiotic relationship between thinking and acting. A more fruitful research methodology will map the spatiotemporal coordinates of the co-constitutive processes that enact, co-temporaneously, the mind and the world to which it is connected. Thinking emerges in ecological space and ecological time from the transactional flow of action and representational opportunities outcropping from a dynamic action-environment interface.

\section{References}

Adams, F., \& Aizawa, K. (2009). Why the mind is still in the head. In M. Aydede \& P. Robbins (Eds.), The Cambridge Handbook of Situated Cognition (pp. 7895). Cambridge: Cambridge University Press.

Ashcraft, M. H., \& Battaglia, J. (1978). Cognitive arithmetic: Evidence for retrieval and decision processes in mental addition. Journal of Experimental Psychology, 4(5), 527-538.

Bakeman, R., \& Quera, V. (2011). Sequential analysis and observational methods for the behavioral sciences. Cambridge ; New York: Cambridge University Press.

Barrett, L. (2011). Beyond the brain: How body and environment shape animal and human minds. Princeton, N.J: Princeton University Press.

Bransford, J. D., \& Franks, J. J. (1971). The abstraction of linguistic ideas. Cognitive Psychology, 2(4), 331350 .

Brighton, H., \& Todd, P. M. (2009). Situating rationality: Ecologically rational decision making with simple heuristics. In P. Robbins \& M. Aydede (Eds.), The Cambridge Handbook of Situated Cognition (pp. 322-346). Cambridge: Cambridge University Press.

Butler, S. C., Berthier, N. E., \& Clifton, R. K. (2002). Two-year-olds' search strategies and visual tracking in a hidden displacement task. Developmental Psychology, 38(4), 581-590. doi:10.1037//00121649.38.4.581

Chemero, A. (2009). Radical embodied cognitive science. Cambridge, Mass.: MIT Press.

Clark, A. (1997). The dynamical challenge. Cognitive Science, 21(4), 461-481.

Clark, A. (2008). Pressing the Flesh: A Tension in the Study of the Embodied, Embedded Mind?*. Philosophy and Phenomenological Research, 76(1), 37-59.

Clark, A. (2010). Material surrogacy and the supernatural: Reflections on the role of artefacts in "off-line" cognition. In L. Malafouris \& C. Renfrew (Eds.), The cognitive life of things: Recasting the boundaries of the mind (pp. 23-28). Cambridge: McDonald Institute for Archaeological Research.

Clark, A., \& Chalmers, D. (1998). The Extended Mind. Analysis, 58(1), 7-19. doi:10.1093/analys/58.1.7

Cowley, S., \& Nash, L. (2013). Language, interactivity and solution probing: Repetition without 
repetition. Adaptive Behavior, 21(3), 187-198. doi: $10.1177 / 1059712313482804$

Finger, S. (1994). Origins of neuroscience: A history of explorations into brain function. New York: Oxford University Press.

Fodor, J. A. (1980). Methodological solipsism considered as a research strategy in cognitive psychology. Behavioral and Brain Sciences, 3(01), 63. doi:10.1017/S0140525X00001771

Gibson, J. J. (1986). The ecological approach to visual perception. Hillsdale, NJ. (Original work published 1979): Erlbaum.

Gick, M. L., \& Holyoak, K. J. (1983). Schema induction and analogical transfer. Cognitive Psychology, 15, 1-38.

Giere, R. N., \& Moffatt, B. (2003). Distributed Cognition: Where the Cognitive and the Social Merge. Social Studies of Science, 33(2), 301-310. doi:10.1177/03063127030332017

Gigerenzer, G., \& Hoffrage, U. (1995). How to improve Bayesian reasoning without instruction: Frequency formats. Psychological Review, 102(4), 684-704. doi:10.1037/0033-295X.102.4.684

Gregory, R. L. (1980). Perceptions as hypotheses. Philosophical Transactions of the Royal Society of London. Series B, Biological Sciences, 290, 181-197.

Henson, R. (2005). What can Functional Neuroimaging Tell the Experimental Psychologist? The Quarterly Journal of Experimental Psychology Section A, 58(2), 193-233. doi:10.1080/02724980443000502

Hollan, J., Hutchins, E., \& Kirsh, D. (2000). Distributed cognition: Toward a new foundation for humancomputer interaction research. ACM Transactions on Computer-Human Interaction, 7, 174-196.

Hutchins, E. (1995). Cognition in the Wild. Cambridge, Mass: MIT Press.

Johnson-Laird, P. N. (1983). Mental models. Cambridge, MA: Harvard University Press.

Kirsh, D. (2013). Embodied cognition and the magical future of interaction design. Avant, 4(2), 124-166. doi:10.12849/40202013.0709.0008

Kirsh, D., \& Maglio, P. (1994). On distinguishing epistemic from pragmatic action. Cognitive Science, 18(4), 513-549. doi:10.1207/s15516709 $\operatorname{cog} 1804 \_1$

Kounios, J., \& Beeman, M. (2009). The Cognitive Neuroscience of Insight, 18(4), 7.
Malafouris, L. (2013). How things shape the mind: A theory of material engagement. Cambridge, Massachusetts: MIT Press.

Marcovitch, S., \& Zelazo, P. D. (1999). The A-Not-B Error: Results from a Logistic Meta-Analysis. Child Development, 70(6), 1297-1313. doi:10.1111/14678624.00095

May, C. J., Schank, J. C., Joshi, S., Tran, J., Taylor, R. J., \& Scott, I. (2006). Rat pups and random robots generate similar self-organized and intentional behavior. Complexity, 12(1), 53-66.

Metcalfe, J., \& Wiebe, D. (1987). Intuition in insight and noninsight problem solving. Memory $\mathcal{E}$ Cognition, 15(3), 238-246. doi:10.3758/BF03197722

Neisser, U. (1967). Cognitive Psychology. New York, NY: Appleton-Century-Crofts [u.a.].

Rupert, R. D. (2009). Cognitive systems and the extended mind. Oxford; New York: Oxford University Press.

Shapiro, L. (2011). Embodied Cognition. New York, NY: Routledge.

Sharpe, T., \& Koperwas, J. (2003). Behavior and sequential analyses. Thousand Oaks, Calif: Sage Publications.

Simon, H. A. (1969). The sciences of the artificial. Cambridge (Mass.); London: The MIT Press.

Smith, L. B., \& Thelen, E. (2003). Development as a dynamic system. Trends in Cognitive Sciences, 7(8), 343-348. doi:10.1016/S1364-6613(03)00156-6

Spivey, M. J., \& Dale, R. (2006). Continuous dynamics in real-time cognition. Current Directions in Psychological Science, 15(5), 207-211. doi:10.1111/j.14678721.2006.00437.x

Steffensen, S. (2013). Human interactivity: Problem-solving, solution probing and verbal patterns in the wild. In S. J. Cowley \& Vallée-Tourangeau, Frederic (Eds.), Cognition beyond the brain: Computation, interactivity and human artifice (pp. 195-221). London: Springer-Verlag.

Thelen, E. (1992). Development as a Dynamic System. Current Directions in Psychological Science, 1, 189193.

Thelen, E., Schöner, G., Scheier, C., \& Smith, L. B. (2001). The dynamics of embodiment: A field theory of infant perseverative reaching. Behavioral and Brain Sciences, 24(1), 1-34. doi:10.1017/S0140525X01003910 
Turing, A. M. (1936). On computable numbers, with an application to the Entscheidungsproblem. Proceedings of the London Mathematical Society, 42(2), 230-265.

Turvey, M., \& Shaw, R. (1999). Ecological foundations of cognition. I: Symmetry and specificity of animalenvironment systems. Journal of Consciousness Studies, 6(11-12), 95-110.

Vallée-Tourangeau, F. (2013). Interactivity, Efficiency, and Individual Differences in Mental Arithmetic. Experimental Psychology, 60(4), 302-311. doi:10.1027/1618-3169/a000200

Vallée-Tourangeau, F., Euden, G., \& Hearn, V. (2011). Einstellung defused: Interactivity and mental set. The Quarterly Journal of Experimental Psychology, 64(10), 1889-1895. doi:10.1080/17470218.2011.605151

Vallée-Tourangeau, F., \& Villejoubert, G. (2013). Naturalising Problem Solving. In S. J. Cowley \& F. ValléeTourangeau (Eds.), Cognition Beyond the Brain (pp. 241-253). London: Springer London.

Van Gelder, T. (1995). What Might Cognition Be, If Not Computation?: Journal of Philosophy, 92(7), 345381. doi:10.2307/2941061

Villejoubert, G., \& Mandel, D. R. (2002). The inverse fallacy:
An account of deviations from Bayes's theorem and the additivity principle. Memory $\mathcal{E}$ Cognition, 30(2), 171-178. doi:10.3758/BF03195278

Villejoubert, G., \& Vallée-Tourangeau, Frederic. (2011). Constructing preferences in the physical world: A distributed-cognition perspective on preferences and risky choices. Frontiers in Psychology, 2. doi:10.3389/fpsyg.2011.00302

Watson, J. D. (1968). The double helix: A personal account of the discovery of the structure of DNA. London: Weidenfeld \& Nicolson.

Weller, A., Villejoubert, G., \& Vallée-Tourangeau, F. (2011). Interactive insight problem solving. Thinking $\mathcal{F}$ Reasoning, 17(4), 424-439. doi:10.1080/13546783.2011.629081

Wilson, A. D., \& Golonka, S. (2013). Embodied Cognition is Not What you Think it is. Frontiers in Psychology, 4. doi:10.3389/fpsyg.2013.00058

Withagen, R., \& Chemero, A. (2012). Affordances and classification: On the significance of a sidebar in James Gibson's last book. Philosophical Psychology, 25(4), 521-537. doi:10.1080/09515089.2011.579424 